\title{
Lewy Body Dementia Without Alzheimer Changes
}

\author{
A.A.F. Sima, A.W. Clark, N.A. Sternberger, L.A. Sternberger
}

\begin{abstract}
Three patients with clinical Alzheimer's disease were found at postmortem examination to have Lewy-bodies and Lewy-like bodies in the cerebral cortex and the pigmented brainstem nuclei. Neuritic plaques were found in neocortical areas but no neurofibrillary tangles. The distribution of cortical neuronal inclusions correlated with the proposed projection of dopamine terminals. Neuronal cell loss was marked in the ventral tegmental area (paranigral nucleus) and the basal nucleus of Meynert, suggesting a defect in dopaminergic and cholinergic innervation of the cerebral cortex. Immunohistochemical investigations revealed positive staining of cortical Lewy- and Lewy-like bodies for monoclonal antibodies to phosphorylated neurofilaments (03-44, 06-17, 04-7). Also cerebral neurons containing no inclusions showed positivity, suggesting an early neurofilament abnormality, preceding the formation of Lewy-type inclusions.
\end{abstract}

RÉSUMÉ: La démence à corps de Lewy en l'absence des changements de type Alzheimer. Des corps de Lewy et des structures similaires aux corps de Lewy ont été retrouvés dans le cortex cérébral et les noyaux pigmentés du tronc cérébral à l'autopsie de trois patients présentant un tableau clinique de maladie d'Alzheimer. Des plaques névritiques ont été trouvées dans les régions corticales, mais pas d'enchevêtrements neurofibrillaires. Il y avait corrélation entre la distribution des inclusions neuronales au niveau du cortex et la projection proposée pour les terminaisons dopaminergiques. La perte cellulaire neuronale était importante au niveau de la région de la décussation ventrale (noyau paranigral) et du noyau basal de Meynert, suggérant un défaut de l'innervation dopaminergique et cholinergique du cortex cérébral. Des études immunohistochimiques ont montré une réaction positive des corps de Lewy et des structures similaires aux corps de Lewy du cortex aux anticorps monoclonaux dirigés contre des neurofilaments phosphorylés (03-44, 06-17, 04-7). Des neurones cérébraux nu contenant pas d'inclusion manifestaient également une réaction positive, suggérant une anomalie neurofilamentaire précoce, précédant la formation des inclusions du type des corps de Lewy.

Can. J. Neurol. Sci. 1986; 13:490-497

Lewy bodies are abnormal perikaryal inclusions of the human brain and of unknown etiology. They are the histopathologic hallmark of Parkinson's disease with predilection sites in the substantia nigra, locus ceruleus and substantia innominata. They are found rarely in the cerebral cortex, but have been reported in association with changes of Alzheimer's disease, ${ }^{1-4}$ Pick's disease, ${ }^{2}$ idiopathic Parkinson's disease without dementia, ${ }^{5-6}$ and in neuroaxonal dystrophies. ${ }^{7-8}$

Ultrastructurally, Lewy bodies are composed of an array of filamentous structures radiating from a dense central core. ${ }^{9}$ Cerebral Lewy bodies or Lewy-like bodies show a conglomerate of less well organized filaments, and the latter lack the central dense core. ${ }^{10}$ Positive immunoreactivity of Lewy bodies with polyclonal and monoclonal antibodies to neurofilaments indicates the presence of neurofilament antigens in these inclusions. "In the present report we describe the quantitative, morphologic and immunohistochemical findings of cortical Lewy bodies and Lewy-like bodies, as well as the results of cell counts of subcortical projection systems in three cases of Lewy body dementia unassociated with Alzheimer's disease.

\section{Case Reports}

\section{Clinical Histories}

\section{Case 1}

This 72 year old male accountant began to notice problems with calculation at age 66. Over the next two years, he experienced forgetfulness and inability to concentrate. On evaluation at age 68 , an electroencephalogram showed diffuse polyrhythmic slow-wave and sharp-wave activity most marked over the frontal and temporal areas. The diagnosis of Alzheimer's disease was made. At the age of 69, he showed mild bradykinesia of gait and he was started on Levodopa. The dementia progressed over the next two years. At age 72 he was admitted in a non-communicative state because of recurrent episodes of aspiration. He died three months later from bronchopneumonia.

From the Section of Neuropathology, Department of Pathology, Health Sciences Centre, Winnipeg, (Dr. Sima); Department of Pathology, Foothills Hospital, Calgary, (Dr. Clark) and the Center for Brain Research, University of Rochester School of Medicine, Rochester, N.Y., (Drs. N.A. Sternberger and L.A. Sternberger)

Reprint requests to: Dr. A.A.F. Sima, Health Sciences Centre, MS459A, 820 Sherbrook Street, Winnipeg, Manitoba, Canada R3A IR9 


\section{Case 2}

This 72 year old female was diagnosed as having Parkinsonism in 1975. Several decades earlier the diagnosis of multiple sclerosis was made. In 1977 she became confined to a wheelchair with weakness and spasms of both legs. She was transferred to a nursing home in 1979. Physical examination in July of 1980 revealed her speech to be incomprehensible. She had difficulty sitting erect and showed spastic weakness of all limbs. Plantar responses were extensor and deep tendon reflexes were increased. Two years later she was noted to be emotionally labile and showed episodes of anger and aggression. She showed bradykinesia and rigidity and was placed on Sinemet with little improvement. Over the ensuing years, she became non-communicative, increasingly stiff, and immobile and was diagnosed as having $\mathrm{Alz}$ heimer's disease. She died from cardiorespiratory arrest in November of 1985.

\section{Case 3}

This 71 year old woman was first admitted in June of 1984 with decreasing appetite and forgetfulness. She displayed a child-like behaviour with angry outbursts. She became disoriented to time and place, appeared weaker and gradually needed more help with ambulation. Neurologic examination was reported as normal. In January of 1985 , she was admitted in a dehydrated state with disorientation to time and place and person. A CT scan showed mild diffuse atrophy of the brain. Over the next 7 months, she showed a rapid deterioration of her mental status and was thought to have Alzheimer's disease. No clinical evaluation was performed during this period. She died three months later from bronchopneumonia.

\section{NeURopathologic Examination}

\section{Methods}

The brains from the 3 cases were fixed in $10 \%$ buffered formalin for at least 10 days. Tissue blocks for light microscopic examination were taken from various parts of the central nervous system. They were embedded in paraffin and stained with hematoxylin and eosin (H \& E), periodic acid-Schiff (PAS), Congo-red, Nissl stain, and Bielschowsky's silver stain. Small pieces of formalin fixed tissue from selected areas were fixed in $2.5 \%$ phosphate-buffered ( $\mathrm{pH} 7.15$ ) glutaraldehyde. They were postfixed in $1 \%$ osmium tetroxide, dehydrated and embedded in Epon. Ultrathin sections were examined electronmicroscopically.
Serial 16um thick sections through paraffin blocks from the basal nucleus of Meynert and the paranigral nucleus were stained with Nissl stain and used for neuronal cell counts. The areas of the basal nucleus of Meynert and the paranigral nucleus were delineated in each section and digitized using a Hewlett-Packard 9874A Digitizer (Hewlett-Packard Company, Fort Collins, Colorado). Only neurons with discernible nuclei were counted in each section. The tissue volume was calculated by adding the products of area and thickness of the serial sections of each nucleus. The cell number was then calculated as neurons per $\mathrm{mm}^{3}$

Forimmunocytochemical examination paraffin sections were incubated with trypsin (GIBCO) at $37^{\circ} \mathrm{C}$ for $10 \mathrm{~min}$. (400 ug/ml) in $0.05 \mathrm{M}$ Tris- $\mathrm{HCl}, \mathrm{pH} 7.6,0.3 \mathrm{M}$ sodium chloride and $0.02 \mathrm{M}$ calcium chloride. The incubated paraffin sections were stained immunocytochemically by using monoclonal first-layer antibodies in a dilution of 1:1000, goat anti-mouse immunoglobulin, and ClonoPAP (peroxidase-monoclonal anti-peroxidase complex) (12). The first-layer antibodies were against phosphorylated neurofilament epitopes (03-44, 06-17, and 04-7) (12).

\section{Gross Examinations}

All three cases showed mild to moderate atrophy of the frontal and temporal areas and moderate ventricular dilatation. The brain weights were 1305,1210 and 1220 grams respectively, in the three cases. Examination of the brain and spinal cord in Case 2 could not confirm the diagnosis of multiple sclerosis.

\section{Lightmicroscopic Findings}

Lightmicroscopic examinations of the three cases revealed similar pathologic findings. The most striking finding was the presence of Lewy bodies and Lewy-like bodies in the pigmented brainstem nuclei and the cerebral cortex. They were found to be most numerous in the entorhinal cortex, the anterior cingulate gyrus, the temporal and frontal cortices, in that order (Table 1). They were found to be concentrated to the 5th and 6th layers of the cortex in most areas, except for the anterior cingulate gyrus and the entorhinal cortex, where they were equally numerous in the 3 rd cortical layer (Figure 1). The

\begin{tabular}{|c|c|c|c|c|c|c|}
\hline \multirow[t]{2}{*}{ Site } & \multicolumn{6}{|c|}{ Pathologic Change } \\
\hline & \multicolumn{3}{|c|}{ Lewy Bodies } & \multicolumn{3}{|c|}{ Lewy-like Bodies } \\
\hline Cerebral Cortex & I & II & III & I & II & 111 \\
\hline Frontal & + & + & + & ++ & + & + \\
\hline Temporal & - & ++ & + & + & $++t$ & + \\
\hline Parietal & + & - & - & ++ & + & + \\
\hline Insular & - & + & + & ++ & ++ & + \\
\hline Cingulate Ant. & ++ & ++ & + & $t+t$ & $+t+$ & + \\
\hline Cingulate Post. & + & + & + & $++t$ & ++ & + \\
\hline Occipital & + & - & - & + & - & - \\
\hline Entorhinal & - & +++ & + & ++ & +++ & + \\
\hline Hippocampus & - & - & - & - & ++ & + \\
\hline Substantia Innominata & ++ & + & + & - & ++ & + \\
\hline Subthalamic Nucleus & - & + & + & - & - & + \\
\hline Amygdala & - & + & + & - & - & + \\
\hline Substantia Nigra & +++ & $+t$ & + & $++t$ & + & + \\
\hline Nucleuṣ Paranigralis & ++ & + & + & + & - & + \\
\hline Locus Ceruleus & + & $+t+$ & + & - & - & $+t+$ \\
\hline Dorsal Raphe Nucleus & + & - & - & + & + & + \\
\hline
\end{tabular}

- No Change; + Small Number ++ Moderate Number; +++ Large Number 

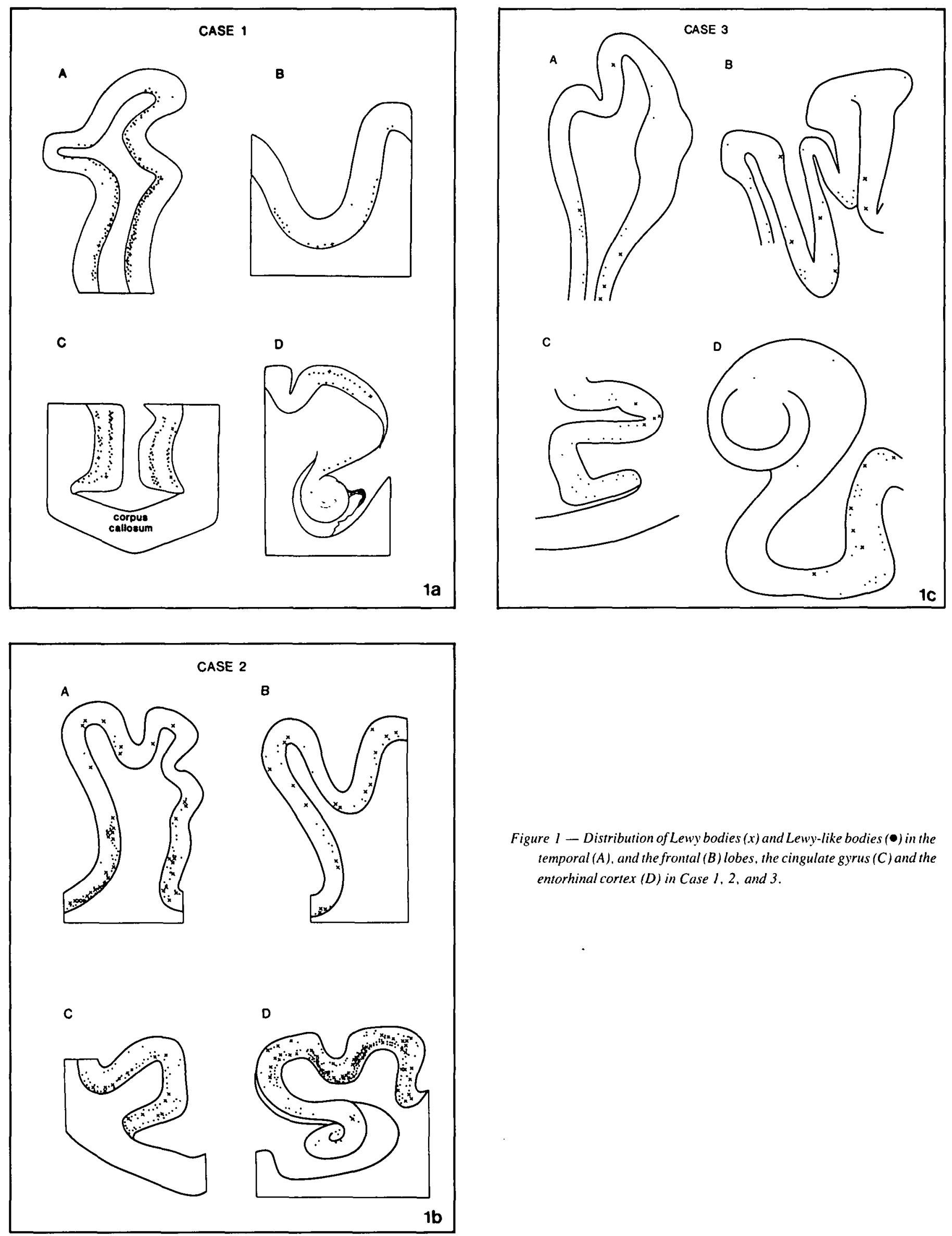

Figure 1 - Distribution of Lewy bodies $(x)$ and Lewy-like bodies $(\bullet)$ in the temporal ( $A)$, and the frontal $(B)$ lobes, the cingulate gyrus $(C)$ and the entorhinal cortex (D) in Case 1, 2, and 3. 
inclusions were found to be most numerous in the depth of the sulci (Figure 1). In areas where Lewy bodies and Lewy-like bodies were numerous, neuronal loss and mild astrogliosis could be demonstrated. A moderate number of senile plaques were demonstrated throughout the neocortex in all three cases, whereas no neurofibrillary tangles were found in neocortical areas in any of the cases (Table 2). Cerebrovascular amyloid deposition was not identified. Lewy-like bodies stained faintly eosinophilic with the H-E stain (Figure 2A) and dark brown to

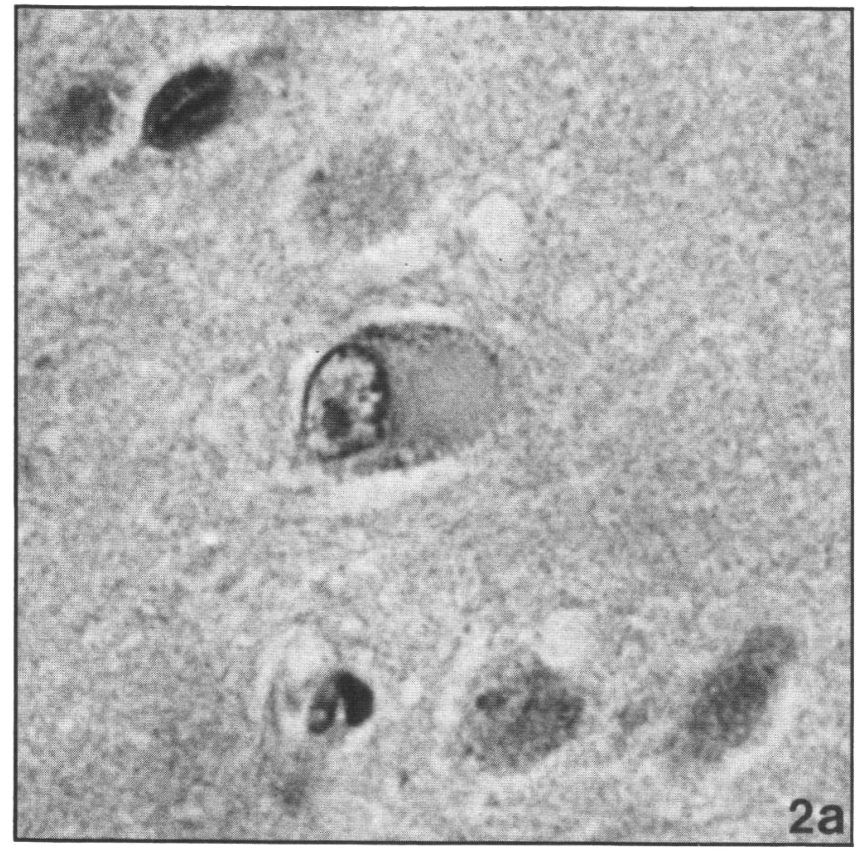

Figure 2(A) - Lewy-like body in the superior temporal gyrus in Case 3 , H.-E., Mag. $870 x$.

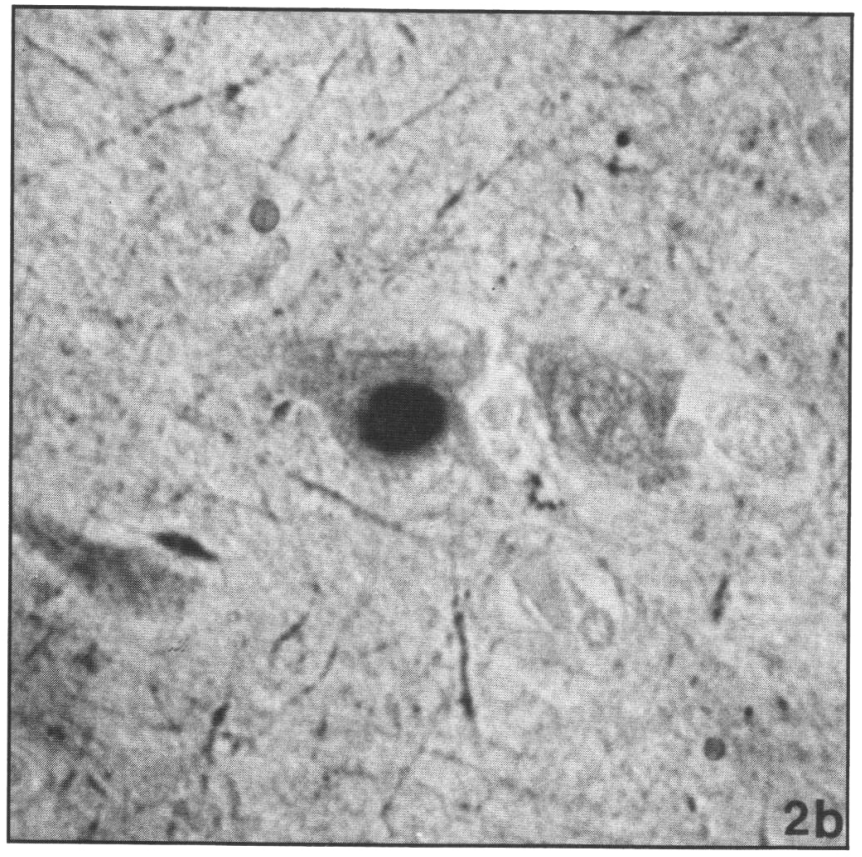

Figure $2(B)$ - Lewy-like body from the same area showing strong reactivity with antibody 06-17 to phosphorylated epitopes. Case 3, Mag 870x. black with Bielschowsky's silver stain. They did not stain with PAS, or Nissl stain. Lewy bodies showed a bright eosinophilic core with the H-E stain (Figure $3 \mathrm{~A}$ ), which also stained more intensely with Bielschowsky's silver stain. The light microscopic staining pattern of the inclusions is summarized in Table 3.

Neuronal cell counts revealed a moderate neuronal loss in the basal nucleus of Meynert (Table 4). The paranigral nucleus of the ventral tegmental area showed marked neuronal loss and gliosis (Figure 4; Table 4).

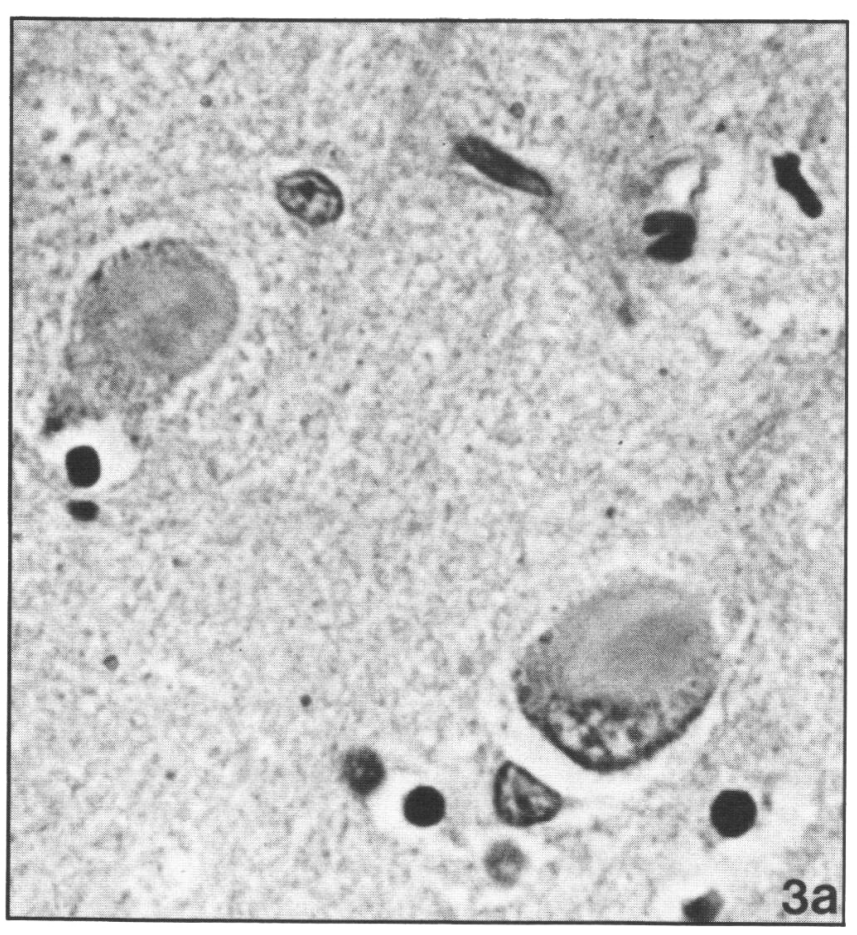

Figure 3(A) - Lewy bodies in the anterior cingulate gyrus in Case 2, H.-E.. Mag 870x.

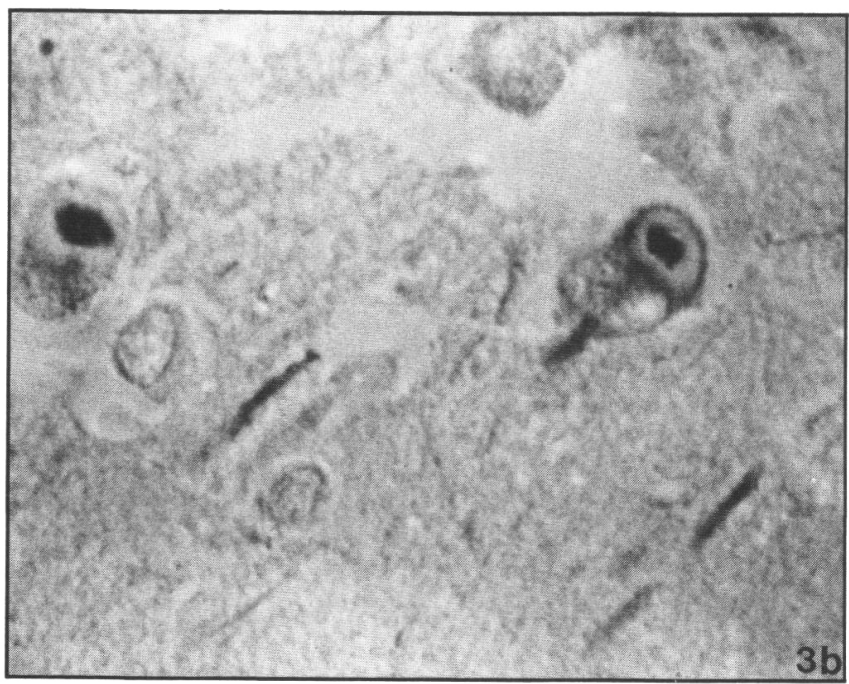

Figure $3(B)$ - Lewy bodies from the same area showing strongly reacting central core and a weakly reacting periphery with antibody $03-44$. This is the reverse staining pattern compared with Lewy bodies in the substantia nigra. (cf. Fig $6 A$ and B) Mag $870 x$. 


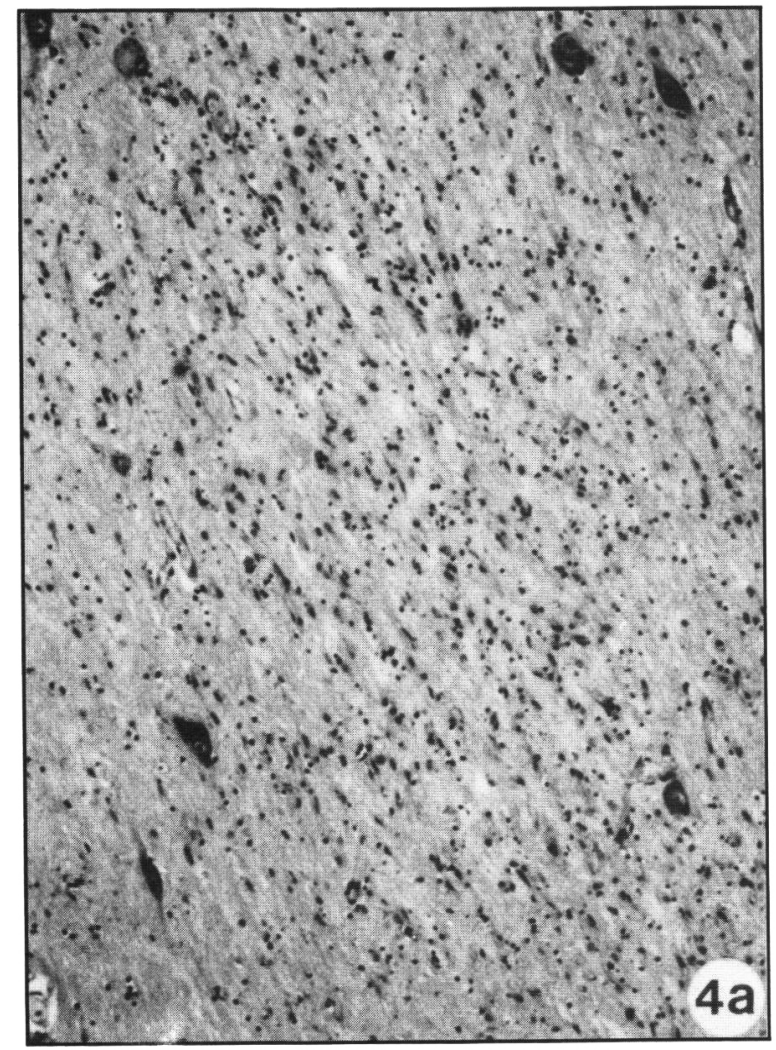

Figure 4(A) - The paranigral nucleus in Case I showing marked neuronal loss and gliosis. This is compared with a normal age. matched subject.

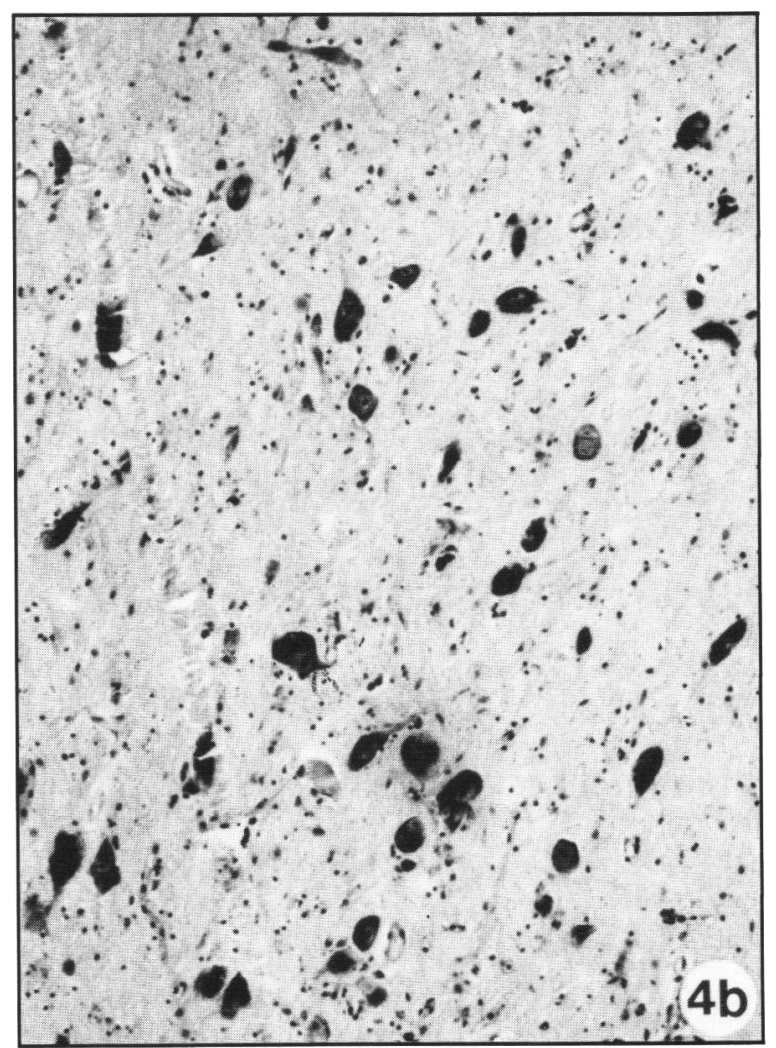

Figure $4(B)-H .-E .$, Mag $120 x$.

\begin{tabular}{|c|c|c|c|c|c|c|}
\hline \multirow[t]{2}{*}{ Site } & \multicolumn{6}{|c|}{ Pathologic Change } \\
\hline & \multicolumn{3}{|c|}{ Neurofibrillary Tangles } & \multicolumn{3}{|c|}{ Senile Plaques } \\
\hline Cerebral Cortex & I & II & III & I & II & III \\
\hline Frontal & - & - & - & $+t$ & ++ & ++ \\
\hline Temporal & - & - & - & ++ & $++t$ & + \\
\hline Parietal & - & - & - & + & + & + \\
\hline Insular & - & - & - & ++ & + & + \\
\hline Cingulate Ant. & - & - & - & + & + & + \\
\hline Cingulate Post. & - & - & - & ++ & ++ & ++ \\
\hline Occipital & - & - & - & + & + & + \\
\hline Entorhinal & - & + & + & ++ & + & ++ \\
\hline Hippocampus & $(+)$ & + & ++ & - & + & ++ \\
\hline Substantia Innominata & - & - & + & + & + & - \\
\hline Subthalamic Nucleus & - & - & - & - & - & - \\
\hline Amygdala & - & - & - & + & + & - \\
\hline Substantia Nigra & + & - & - & - & - & - \\
\hline Nucleus Paranigralis & - & - & - & - & - & - \\
\hline Locus Ceruleus & - & - & - & - & - & - \\
\hline Dorsal Raphe Nucleus & + & - & - & - & - & - \\
\hline
\end{tabular}

- No Change; + Small Number ++ Moderate Number +++ Large Number

Table 3: Light microscopic staining properties of Lewy bodies and Lewy-like bodies

\begin{tabular}{lccccc}
\hline \hline & H \& E & PAS & $\begin{array}{c}\text { Bielschowsky's } \\
\text { Silver Stain }\end{array}$ & $\begin{array}{c}\text { Nissl } \\
\text { Stain }\end{array}$ & $\begin{array}{c}\text { Congo } \\
\text { Red }\end{array}$ \\
\hline Lewy-Body & ++ & - & + & - & - \\
Lewy-like Body & + & - & +++ & - & - \\
\hline
\end{tabular}

- Negative; + Weakly Positive;

++ Moderately Positive; +++ Strongly Positive.
Table 4: Neuronal counts $\left(/ \mathrm{mm}^{3}\right)$ in the paranigral nucleus and the basal nucleus of Meynert in Cases 1, 2 and 3 compared with 2 age-matched control subjects.

\begin{tabular}{lcc}
\hline \hline & P.N. & BnM \\
\hline Controls & & \\
(Age-Matched) & & \\
$(\mathrm{N}=2)$ & $2400 \pm 141$ & $1814 \pm 212$ \\
Case 1 & 242 & 750 \\
Case 2 & 225 & 711 \\
Case 3 & 538 & 1107 \\
\hline
\end{tabular}




\section{Electronmicroscopic Findings}

The cortical Lewy-like bodies consisted of both granular and filamentous material. Lewy bodies showed a variably increased density of the central zone. The filaments measured $10-18 \mathrm{~nm}$ in both types of inclusions. The radial arrangement of peripheral filaments typical of brainstem Lewy bodies was less pronounced (Figure 5).

\section{Immunohistochemical Findings}

Three monoclonal antibodies (06-17, 03-44, 04-7) directed against phosphorylated neurofilament epitopes were employed. Lewy-like bodies of the substantia nigra showed weak reactivity (Figure 6A). Lewy bodies of the same nucleus showed a weakly reacting central zone and a strongly reacting peripheral ring (Figure 6B). A similar pattern was occasionally seen in cortical Lewy and Lewy-like bodies. Most commonly, however, Lewy-like bodies showed a strong reactivity with antibodies to phosphorylated epitopes of neurofilaments (Figure 2B). Cortical Lewy bodies showed strong reactivity of the central core and weak or no reactivity of the periphery (Figure 3B). Neurones of the 5th cortical layer containing no identifiable inclusions often revealed a diffuse positive immunostaining with monoclonal antibodies to phosphorylated neurofilaments (Figure 7). The immunoreactivity of Lewy bodies and Lewy-like bodies for the three monoclonal antibodies is summarized in Table 5.

\section{Discussion}

Alzheimer type changes were found in the present 3 cases, consistent with the advanced age of the patients, but with a distribution that did not warrant the diagnosis of Alzheimer's disease.

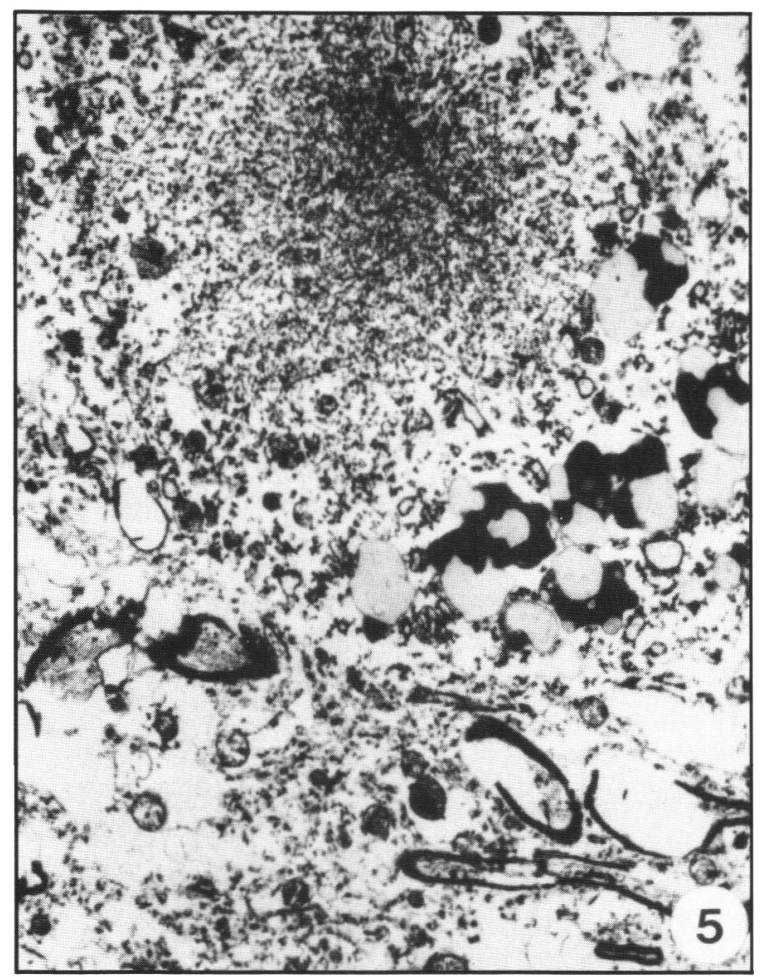

Figure 5 - Electron micrograph of a Lewy-like body in the entorhinal cortex from Case 1. It is made up of neuro-filaments measuring $10-18 \mathrm{~nm}$ in diameter. No parahelical filaments were identified. Mag 8,100x.
Lewy body dementia without concomitant histopathologic changes of Alzheimer's disease is a seldom recognized entity. ${ }^{13-14}$ In the present cases, cerebral Lewy bodies and Lewylike bodies showed a distributional pattern not previously described. In the neocortex the Lewy-type inclusions were confined to the deeper layers 5 and 6 , whereas in the anterior cingulate gyrus and in the entorhinal cortex, inclusions were also widely distributed in the 3rd cortical layer. This distribution of Lewy bodies and Lewy-like bodies correlates well with the proposed mesocortical dopaminergic projections to the cerebral cortex. ${ }^{15.16}$ The dopaminergic fibers projecting to the

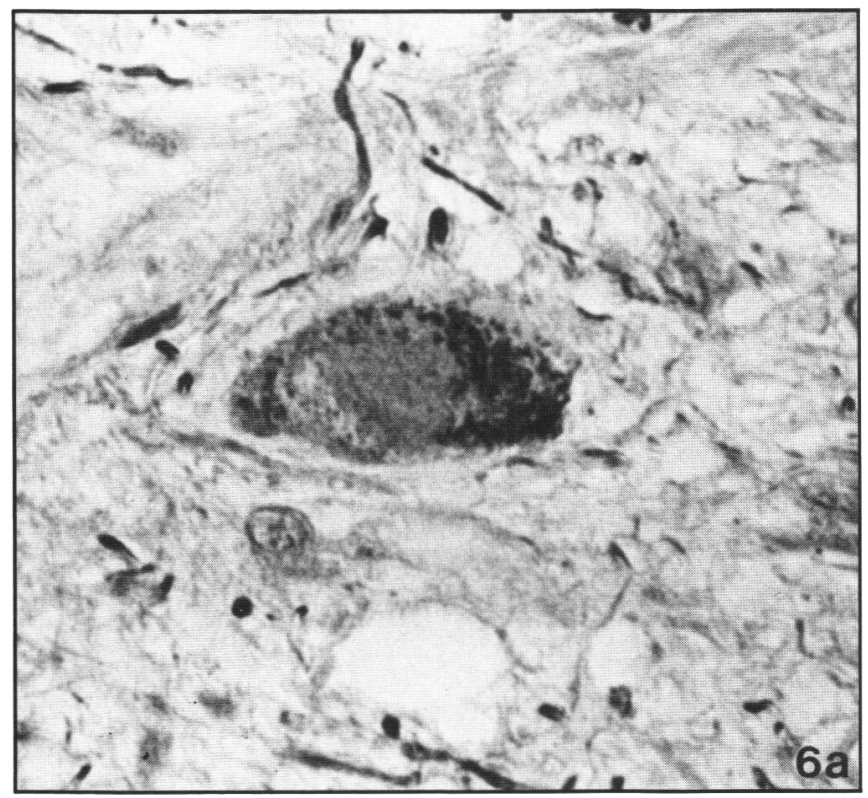

Figure $6(A)-L e w y$-like inclusion in the substantia nigra in Case 3, showing no reactivity with antibody $03-44$.

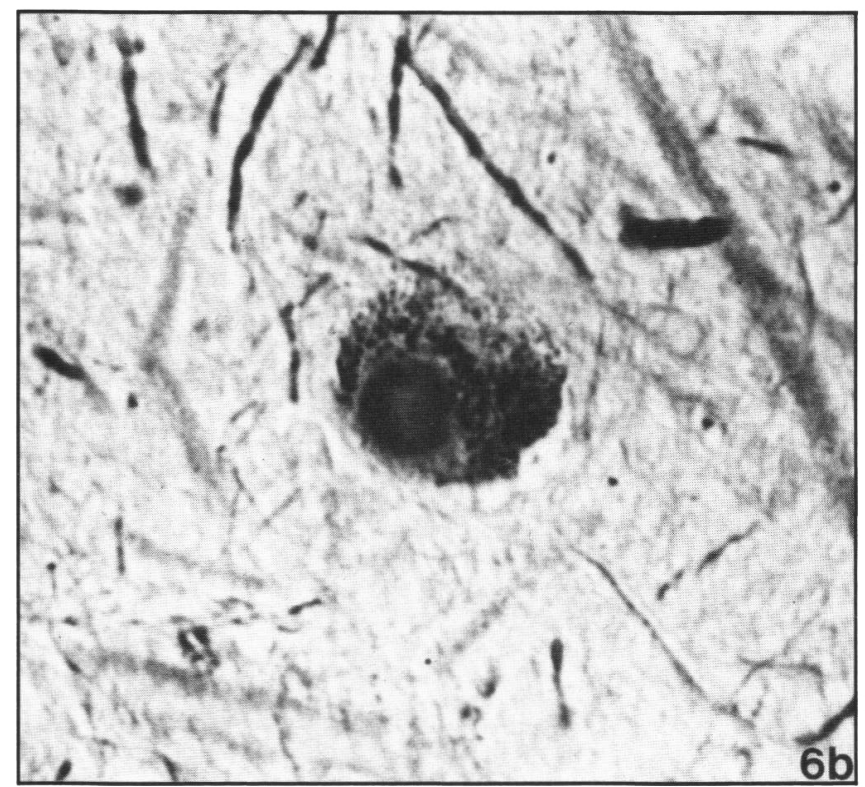

Figure $6(B)$ - Lewy-body in the same nucleus showing strong reactivity of the periphery with antibody $03-44$, and weak reactivity of the central core. Compare these patterns of reactivity with those of cerebral Lewy-type inclusions. (Fig $3 A$ and B), Mag 870x. 
cerebral cortex originate from the ventral tegmental area, mainly from the paranigral nucleus and to a lesser extent from locus ceruleus (Figure 8). ${ }^{16}$ They terminate most frequently in the layers 5 and 6 of the frontal cortex, whereas the projections to the anterior cingulate and ventral entorhinal areas terminate in the 2 nd and 3 rd layers as well. ${ }^{15-17}$

In the present cases the basal nucleus of Meynert showed a moderate neuronal loss, whereas the paranigral nucleus showed a $90 \%$ neuronal loss in Case 1 and 2 and somewhat less in Case 3 , suggesting a substantial diminution of cortical dopaminergic input.

It is possible that dopaminergic and cholinergic deafferentation of the cortical neuronal population may underlie not only the intellectual impairment and cognitive defects observed in the present patients but also the histopathologic findings of Lewy-type inclusions. ${ }^{16}$ In other systems such as the inferior olivary nucleus and the substantia nigra, abnormal neurofilament accumulations are thought to represent a transsynaptic reaction. ${ }^{18-20}$

The ultrastructural features and dimensions of the neurofilaments constituting the inclusions were similar to those described in brainstem Lewy bodies. ${ }^{21}$

Immunohistochemical investigations using monoclonal antibodies to phosphorylated neurofilaments revealed positive staining of Lewy-like bodies and of the central core in Lewy bodies of the cortices. This is different from the staining pattern of brainstem Lewy bodies in which phosphorylated neurofilaments are found in the peripheral rim of Lewy bodies. ${ }^{21}$

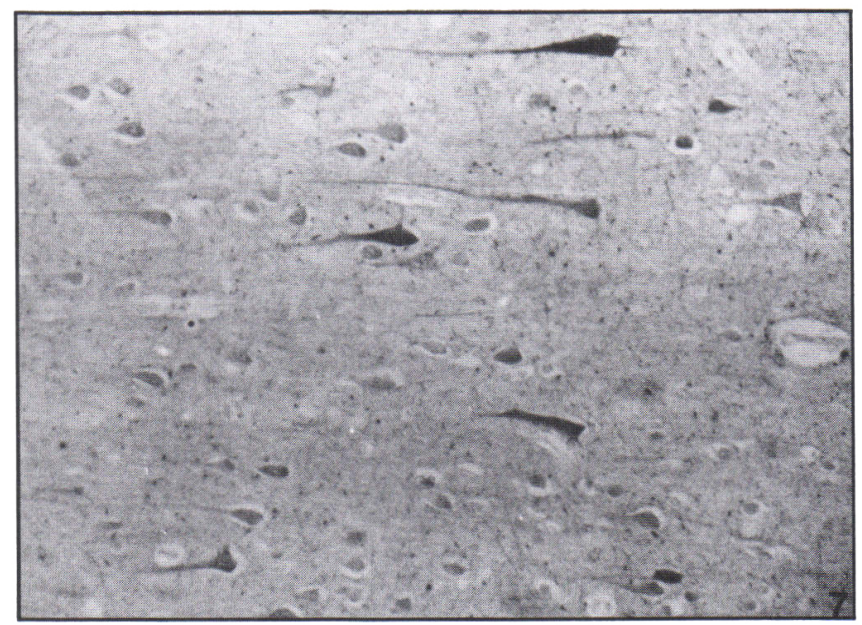

Figure 7 - Positive immunostaining with antibody 06-17 of neurons devoid of inclusions in the 5th cortical layer of the frontal cortex. Case 2, Mag $104 x$
Neurons of the same cortical layers containing no inclusions showed abnormal phosphorylated neurofilaments, not seen in normal controls, suggesting an early post-translational phosphorylation of neurofilaments preceding the formation of Lewy type inclusions.

Based on the pathology of the present three cases, we propose that Lewy body dementia without Alzheimer's disease may be a primary defect in mesocortical dopaminergic projections resulting in a transsynaptic abnormal neurofilamentous reaction of the target neurons, expressed by the presence of Lewy-like bodies and Lewy bodies.

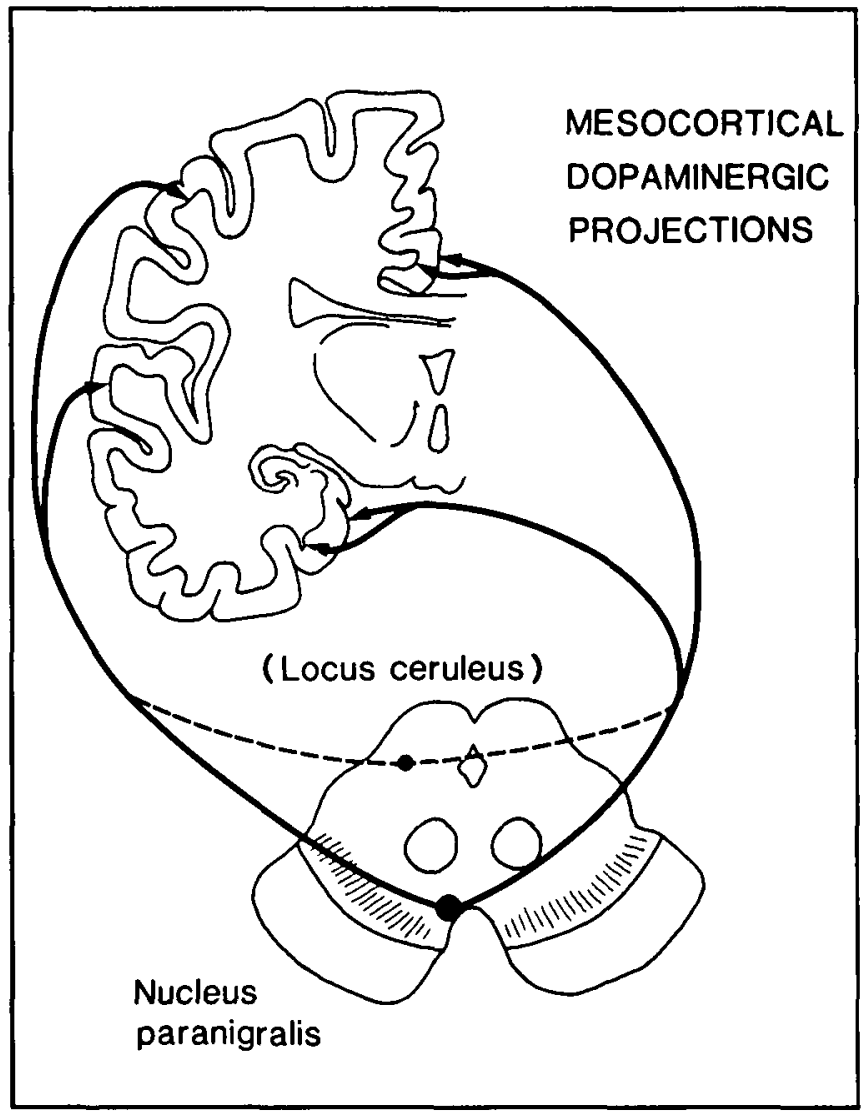

Figure 8 - Schematic illustration of proposed mesocortical dopaminergic projections. Most dopaminergic fibers originate from the paranigral nucleus and project to layers 5 and 6 of the temporal, frontal and parietal cortices, and to layers 5-6 and 3 in the entorhinal and anterior cingulate cortices. These projections correspond to the distribution of Lewy bodies and Lewy-like bodies in the present cases (cf. Fig I).

Table 5: Immunoreactivity to phosphorylated neurofilaments.

\begin{tabular}{lccccc}
\hline \hline & & \multicolumn{2}{c}{ Lewy Bodies } & \multicolumn{2}{c}{ Lewy-Like Bodies } \\
Antibodies & Central Core & Periphery & Central Core & Periphery & Substantia Nigra \\
\hline $06-17$ & - & ++ & +++ & + & - \\
$03-44$ & + & +++ & ++ & - & - \\
$04-7$ & + & ++ & ++ & + & +++ \\
\hline
\end{tabular}

- Negative; + Weak; ++ Moderate; and +++ Strong Reactivity 


\section{ACKNOWLEDGEMENT}

The authors are indebted to Mrs. Jackie McKane for preparing the manuscript.

\section{REFERENCES}

1. Woodard JS. Concentric hyalin inclusion body formation in mental disease. Analysis of 27 cases. J Neuropath Exp Neurol 1962;21: 442-449.

2. Kosaka K, Oyanagi S, Matsushita M, et al. Presenile dementia with Alzheimer-, Pick- and Lewy body changes. Acta Neuropathol (Berl) 1976; 36: 221-233.

3. Forno LS, Barbour PJ, Norville RL. Presenile dementia with Lewy bodies and neurofibrillary tangles. Arch Neurol 1978; 35: 818-822.

4. Kosaka K, Yoshimura M, Ikeda K, et al. Diffuse type of Lewy body disease: progressive dementia with abundant cortical Lewy bodies and senile changes of varying degree - a new disease? Clin Neuropath 1984; 3: 185-192.

5. Yagashita S, Itoh $Y$, Amano N, et al. Atypical senile dementia with widespread Lewy-type inclusion in the cerebral cortex. Acta Neuropath. (Berl) 1980; 49: 187-191.

6. Yoshimura M. Cortical changes in the Parkinsonian brain: a contribution to the delineation of "diffuse Lewy body disease". J Neurol 1983; 229: 17-32.

7. Vuia O. Neuroaxonal dystrophy, a juvenile adult form. Clin Neurol Neurosurg 1977; 79: 305-315.

8. Williamson K, Sima AAF, Curry B, Ludwin SK. Neuroaxonal dystrophy in young adults: A clinicopathologic study of two unrelated cases. Ann Neurol 1982; 11:335-343.

9. Duffy PE, Tennyson UM. Phase and electron microscopic observations of Lewy bodies and melanin granules in the substantia nigra and locus coeruleus in Parkinson's disease. J Neuropath Exp Neurol 1965; 24: 398-414.
10. Itoh T, Momma Y, Ogasawara N. An electron microscopic study on atypical presenile dementia with numerous Lewy bodies in the cerebral cortex. Folia Psychiatr Neurol Jpn 1982; 36: 99-106.

11. Goldman JE, Yen SH, Chiu F-C, Peress NS. Lewy bodies of Parkinson's disease contain neurofilament antigen. Science 1983; 221: 1082-1084.

12. Sternberger LA, Sternberger NA. Monoclonal antibodies distinguish phosphorylated and nonphosphorylated forms of neurofilaments in situ. Proc Natl Acad Sci USA, 1983; 80: 6126-6130.

13. Ikeda K, Hori A, Bode G. Progressive dementia with "diffuse Lewy-type inclusions"' in cerebral cortex. A case report. Arch Psychiatr Nervenkr 1980; 228: 243-248.

14. Eggertson DE, Sima AAF. Dementia with cerebral Lewy bodies. A mesocortical dopaminergic defect? Arch Neurol 1986; 43: 524-527.

15. Lindvall O, Bjorklund A, Divac I. Organization of mesencephalic dopamin neurons projecting to neocortex and septum. In Costa E and Gessa, GL eds. Advances in Biochemical Psychopharmacology Vol. 16, New York, Raven Press, New York, 1977, pp 39-46.

16. Javoy-Agid F, Agid Y. Is the mesocortical dopaminergic system involved in Parkinson disease? Neurology 1980; 30: 1326-1330.

17. Lindvall O, Bjorklund A, Moore RY, et al. Mesencephalic dopamine neurons projecting to the neocortex. Brain Res 1974; 81 : 325-331.

18. Horoupian DS, Wisniewski H. Neurofilamentous hyperplasia in inferior olivary hypertrophy. J Neuropath Exp Neurol 1971; 30: 571-582.

19. Forno LS. Reaction of the substantia nigra to massive basal ganglia infarction. Acta Neuropath (Berl) 1983 62: 96-102.

20. Yagishita S, Itoh $Y$, Nakano T. Hypertrophy of the olivary nucleus. An ultrastructural study. Acta Neuropath (Berl) 1986 69: 132-138.

21. Forno LS, Sternberger LA, Sternberger NA, et al. Reaction of Lewy bodies with antibodies to phosphorylated and non-phosphorylated neurofilaments. Neuroscience Letter 1986; 64: 253-258. 